\title{
OPEN Hydroa vacciniforme-like lymphoproliferative disorder in Korea
}

\author{
Byeol Han ${ }^{1,2}$, Keunyoung Hur ${ }^{1}$, Jungyoon Ohn ${ }^{1,2}$, Tae Min Kim ${ }^{3,4}$, Yoon Kyung Jeon ${ }^{4,5}$, \\ You Chan Kim ${ }^{6}$ \& Je-Ho Mun ${ }^{1,2}$
}

Hydroa vacciniforme-like lymphoproliferative disorder (HVLPD) is a rare Epstein-Barr virus (EBV)associated lymphoproliferative disease. The disease course of HVLPD varies from an indolent course to progression to aggressive lymphoma. We investigated the characteristics of HVLPD in Korean patients. HVLPD patients at Seoul National University Hospital between 1988 and 2019 were retrospectively analyzed. This study included 26 HVLPD patients who all presented with recurrent papulovesicular and necrotic eruption on the face, neck, and extremities. EBV was detected from the skin tissues of all patients. HVLPD was diagnosed during childhood (age $<18$ years) in seven patients (26.9\%) and in adulthood (age $\geq 18$ years) in 19 cases $(73.1 \%)$. The median age at diagnosis was 24.0 years (range 7-70 years). HVLPD has various clinical courses, from an indolent course to progression to systemic lymphoma. Fourteen patients $(53.8 \%)$ developed lymphoma: systemic EBVpositive T-cell lymphoma $(n=9,34.6 \%)$; extranodal natural killer/T-cell lymphoma, nasal type $(n=3$, $11.5 \%)$; aggressive natural killer/T-cell leukemia $(n=1,3.8 \%)$; and EBV-positive Hodgkin lymphoma $(n=1,3.8 \%)$. Mortality due to HVLPD occurred in five patients (26.3\%) in the adult group, while it was one patient (14.3\%) in the child group. As lymphoma progression and mortality occur not only in childhood but also in adulthood, adult-onset cases may need more careful monitoring.

Hydroa vacciniforme-like lymphoproliferative disorder (HVLPD) is a rare Epstein-Barr virus (EBV)-associated T-cell or natural killer (NK)-cell lymphoproliferative disease. According to the revised 4th World Health Organization (WHO) classification, the term HVLPD has been renamed from hydroa vacciniforme-like lymphoma to HVLPD owing to its relationship to chronic active EBV infection (CAEBV) and the broad spectrum of its clinical course $^{1}$. Although HVLPD usually affects children, adult and elderly cases have been reported ${ }^{2}$. Clinically, it is characterized by recurrent erythematous papules and vesicles that leave pitted scars on sun-exposed areas, including the face and forearms. The disease course of HVLPD varies from an indolent course to systemic lymphoma. Our experience and the literature show that mortality or a fatal outcome from the progression of HVLPD to lymphoma can also occur in adults ${ }^{3-6}$. In the present study, we report on 26 cases of HVLPD encountered at our hospital during the last three decades.

\section{Results}

Clinical features. A total of 26 cases were enrolled with diagnosis of HVLPD based on clinical and histopathologic features between 1988 and 2019 in the Department of Dermatology of Seoul National University Hospital (SNUH). Fifteen cases had been previously reported ${ }^{7-13}$. There were seven children (age $<18$ years) and 19 adults (age $\geq 18$ years), with a median age of 24.0 years (range $7-70$ years) at the time of diagnosis. The age at which the skin lesions of HVLPD first appeared varied, with a median age of 17.5 years (range 3-70 years). Among 19 adult patients, $6(31.6 \%)$ reported that they had skin lesions in childhood. There were 16 male patients and 10 female patients (male-to-female ratio 1.6:1). All patients had recurrent vesicles, papules, crusted papulovesicles, necrotic ulcers, and pitted scars on sun-exposed areas (Fig. 1 and Tables 1 and 2). Besides lesions on the face and arms, skin lesions on non-sun-exposed areas, including the trunk, were present in seven patients

\footnotetext{
${ }^{1}$ Department of Dermatology, Seoul National University College of Medicine, Seoul, Republic of Korea. ${ }^{2}$ Institute of Human-Environment Interface Biology, Seoul National University, Seoul, Republic of Korea. ${ }^{3}$ Department of Internal Medicine, Seoul National University Hospital, Seoul, Republic of Korea. ${ }^{4}$ Seoul National University Cancer Research Institute, Seoul, Republic of Korea. ${ }^{5}$ Department of Pathology, Seoul National University College of Medicine, Seoul, Republic of Korea. ${ }^{6}$ Department of Dermatology, Ajou University School of Medicine, Suwon, Republic of Korea. ${ }^{\circledR}$ email: jehomun@gmail.com
} 


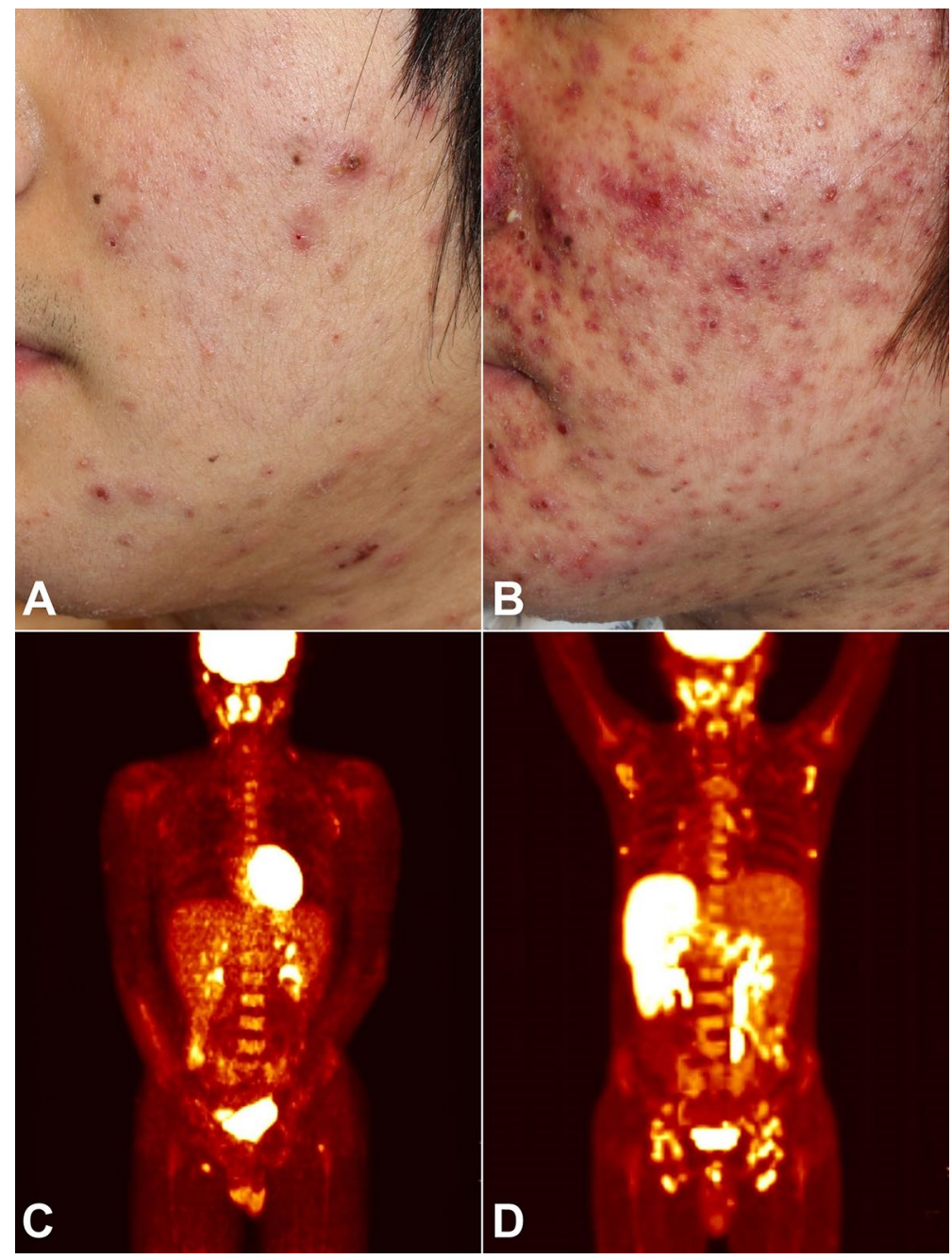

Figure 1. An 18-year-old adult (patient no. 8) diagnosed with hydroa vacciniforme-like lymphoproliferative disease that progressed to systemic Epstein-Barr virus-positive (EBV +) T-cell lymphoma. (A) Erythematous crusted necrotic papulovesicles on the face. (B) Six months after the diagnosis, the skin lesions became aggravated. At this stage, systemic EBV+ T-cell lymphoma was diagnosed. (C) In the positron emission tomography-computed tomography (PET-CT) scan, there was no evidence of systemic involvement at the first visit. (D) However, as the skin eruption deteriorated (B), the PET-CT scan revealed multiple hypermetabolic lesions in the cervical, axillary, abdominal, and inguinal lymph nodes and in the spleen.

(26.9\%). Oral ulcer was noted in nine patients $(34.6 \%)$, and facial swelling was observed in three patients $(11.5 \%)$. At the first consultation, six patients $(23.1 \%)$ had fever and cervical lymphadenopathy and two patients (7.7\%) had splenomegaly. The median follow-up duration was 36.0 months (range 12-216 months). Ultraviolet (UV) A radiation provocation test was performed in seven patients. Among them, five patients (71.4\%) had skin eruption with papulovesicles. Mosquito bite hypersensitivity was noted in three patients (11.5\%). Serum EBV viral load was evaluated in 11 patients $(42.3 \%)$. All patients showed serum EBV. High levels of viral load, ranging from 54,275 to 47,925,563 copies/mL (median 1,784,901 copies/mL), were observed during follow-up.

Histopathologic features, immunohistochemical findings, in situ hybridization, and molecular studies. The histopathologic features of cutaneous tissue were similar in all patients. Dense and diffuse infiltration of atypical lymphocytes was seen in the dermis and subcutaneous fat (Fig. 2). Epidermal necrosis, vesiculation, subepidermal edema, and perivascular and periappendageal atypical lymphocytic infiltration were found. The proliferated atypical lymphocytes were of small to intermediate size, with enlarged, round to oval nuclei. There were an inflammatory background with lymphocytes, eosinophils, histiocytes and plasma cells. The immunohistochemistry results and molecular study findings are summarized in Table 2. EBV-encoded small nonpolyadenylated RNA (EBER) was positive in all patients. EBER-positive cells were predominantly seen in the perivascular and periappendageal area in the dermis and subcutaneous fat. Detection of monoclonal $\mathrm{T}$-cell receptor $\gamma$ gene rearrangements was performed in 13 patients. Among them, four patients (30.8\%) showed these rearrangements. 


\begin{tabular}{|c|c|}
\hline Variable & Total $\mathrm{N}=\mathbf{2 6}, \mathrm{n}(\%)$ \\
\hline Age at diagnosis, mean \pm SD (range) (years) & $25.3 \pm 12.8(7-70)$ \\
\hline Children $(<18)$ & $7(26.9)$ \\
\hline Adult $(\geq 18)$ & $19(73.1)$ \\
\hline Age at onset, mean \pm SD (range) (years) & $19.8 \pm 14.9(3-70)$ \\
\hline During childhood $(<18)$ & $13(50.0)$ \\
\hline During adulthood $(\geq 18)$ & $13(50.0)$ \\
\hline Disease duration, mean \pm SD (range) (years) & $5.5 \pm 5.2(0-17)$ \\
\hline \multicolumn{2}{|l|}{ Sex } \\
\hline Male & $16(61.5)$ \\
\hline Female & $10(38.5)$ \\
\hline \multicolumn{2}{|l|}{ Anatomic location of cutaneous manifestations } \\
\hline Sun-exposed area & $26(100)$ \\
\hline Non-sun-exposed area & $7(26.9)$ \\
\hline \multicolumn{2}{|l|}{ Extracutaneous involvement } \\
\hline Yes & $16(61.5)$ \\
\hline No & $10(38.5)$ \\
\hline \multicolumn{2}{|l|}{ UVA provocation test $[n=7]^{a}$} \\
\hline Positive & $5(71.4)$ \\
\hline Negative & $2(28.6)$ \\
\hline Serum EBV DNA load, mean \pm SD (range) $($ copies $/ \mathrm{mL})[\mathrm{n}=11]^{\mathrm{b}}$ & $9,849,887.6 \pm 15,824,779.4(54,275-47,925,563)$ \\
\hline \multicolumn{2}{|c|}{ Development of systemic lymphoma } \\
\hline Yes & $14(53.8)$ \\
\hline Systemic EBV+ T-cell lymphoma & $9(34.6)$ \\
\hline Extranodal NK/T-cell lymphoma, nasal type & $3(11.5)$ \\
\hline Aggressive NK/T-cell leukemia & $1(3.8)$ \\
\hline EBV+ Hodgkin lymphoma & $1(3.8)$ \\
\hline No & $12(46.2)$ \\
\hline \multicolumn{2}{|l|}{ Outcome } \\
\hline Alive with disease & $19(73.1)$ \\
\hline Mortality due to the disease & $6(23.1)$ \\
\hline Mortality due to another disease & $1(3.8)$ \\
\hline
\end{tabular}

Table 1. Summary of characteristics of patients with hydroa vacciniforme-like lymphoproliferative disease. $E B V+$ Epstein-Barr Virus-positive, $N K$ natural killer, $S D$ standard deviation, UVA ultraviolet A. ${ }^{a} U V A$ provocation test was performed in seven patients. ${ }^{b}$ Serum EBV DNA load was evaluated in 11 patients.

Treatment and disease course. Various treatments were applied. Sun exposure avoidance was recommended to all patients. Systemic steroid was used to control the disease in 14 patients (53.8\%). Systemic acyclovir or minocycline was administered in three patients. Thirteen patients received chemotherapy as the first-line or second-line therapy because of disease activity exacerbation. Seven patients died during the follow-up (mortality rate $26.9 \%$ ), six of whom died of the disease; one of whom (case no. 17) died of breast cancer. Therefore, HVLPD mortality occurred in seven patients $(23.1 \%)$.

In the child group, one patient showed remission (14.3\%) and three patients had partial remission and recurrence $(42.9 \%)$. The disease progressed to systemic lymphoma or leukemia in three patients (42.9\%), two of whom had a diagnosis of systemic EBV-positive (EBV+) T-cell lymphoma of childhood and one of whom had aggressive NK/T-cell leukemia. The mortality rate of HVLPD in the child group was $14.3 \%$ (1/7). The cause of death was aggressive NK/T-cell leukemia.

In the adult group, remission was noted in two patients (10.5\%) and partial remission was observed in six patients (31.6\%). Eleven cases (57.9\%) were associated with systemic lymphoma, of which seven (36.8\%) progressed to systemic EBV+ T-cell lymphoma; 3 (15.8\%) to extranodal NK/T-cell lymphoma, nasal type; and 1 (5.3\%) to EBV+ Hodgkin lymphoma. The mortality rate of HVLPD in the adult group was $31.6 \%$ (6 of 19). When we excluded the case of breast cancer death, the rate of disease-specific death was $26.3 \%$ (5 of 19) in the adult group. The causes of death were progression to systemic EBV+ T-cell lymphoma in four patients and extranodal NK/T-cell lymphoma, nasal type, in one patient.

Progression to lymphoma and mortality in relation to age at diagnosis, disease onset, and disease duration. We compared the rates of HVLPD progression to lymphoma and disease-related mortality between patients diagnosed during childhood and those diagnosed during adulthood. Although the rates of both were higher among those diagnosed during adulthood, the differences were not statistically significant $(p=0.665$ 


\begin{tabular}{|c|c|c|c|c|c|c|c|c|c|c|c|}
\hline Patient no. & Sex & \begin{tabular}{|l|} 
Age at \\
diagnosis, \\
years (age at \\
onset, years)
\end{tabular} & $\begin{array}{l}\text { Cutaneous } \\
\text { manifestations }\end{array}$ & \begin{tabular}{|l|} 
IHC, \\
EBER-1 ISH, \\
TCR $\gamma$ gene \\
rearrangement
\end{tabular} & $\begin{array}{l}\text { Serum EBV } \\
\text { DNA load } \\
\text { (copies/mL) }\end{array}$ & $\begin{array}{l}\text { UVA } \\
\text { provocation } \\
\text { test }\end{array}$ & $\begin{array}{l}\text { Extracutaneous } \\
\text { involvement }\end{array}$ & Treatment & $\begin{array}{l}\text { Disease } \\
\text { course }\end{array}$ & $\begin{array}{l}\text { Follow-up } \\
\text { (months) }\end{array}$ & $\begin{array}{l}\text { Last status } \\
\text { during } \\
\text { follow-up }\end{array}$ \\
\hline 1 & $\mathrm{~F}$ & $7(6)$ & $\begin{array}{l}\text { Erythematous } \\
\text { crusted papu- } \\
\text { lovesicles on the } \\
\text { face and oral } \\
\text { ulcer }\end{array}$ & $\begin{array}{l}\text { CD3+, CD20+a } \\
\text { few, Ki-67 10\%, } \\
\text { EBV ISH+ }\end{array}$ & $15,462,763$ & Positive & None & Observation & $\begin{array}{l}\text { Partial } \\
\text { remission } \\
\text { and recur- } \\
\text { rence }\end{array}$ & 72 & AWD \\
\hline 2 & M & $10(9)$ & $\begin{array}{l}\text { Erythematous } \\
\text { crusted papu- } \\
\text { lovesicles on } \\
\text { the face }\end{array}$ & $\begin{array}{l}\text { CD3+, CD20+ } \\
\text { a few, CD56-, } \\
\text { EBV ISH+ }\end{array}$ & ND & Negative & None & $\begin{array}{l}\text { Systemic } \\
\text { steroid, } \\
\text { minocycline }\end{array}$ & $\begin{array}{l}\text { Partial } \\
\text { remission } \\
\text { and recur- } \\
\text { rence }\end{array}$ & 216 & AWD \\
\hline $3^{7}$ & $\mathrm{~F}$ & $11(5)$ & $\begin{array}{l}\text { Erythematous } \\
\text { crusted papu- } \\
\text { lovesicles on the } \\
\text { face and arm, } \\
\text { and oral ulcer }\end{array}$ & $\begin{array}{l}\text { CD3+, } \\
\text { CD45RO+, } \\
\text { CD8+, CD20-, } \\
\text { CD56+ few, } \\
\text { LMP-1-, EBV } \\
\text { ISH+ }\end{array}$ & $1,784,901$ & ND & None & $\begin{array}{l}\text { Systemic } \\
\text { steroid on } \\
\text { recurrence }\end{array}$ & $\begin{array}{l}\text { Partial } \\
\text { remission } \\
\text { and recur- } \\
\text { rence }\end{array}$ & 180 & AWD \\
\hline $4^{8}$ & F & $12(3)$ & $\begin{array}{l}\text { Erythematous } \\
\text { crusted papu- } \\
\text { lovesicles on } \\
\text { the face, arm, } \\
\text { and leg }\end{array}$ & \begin{tabular}{|l|} 
CD3+, \\
CD45RO+, \\
CD56-, S-100-, \\
lysozyme+focal, \\
EBV ISH+
\end{tabular} & ND & ND & $\begin{array}{l}\text { Liver, spleen, } \\
\text { bone marrow }\end{array}$ & $\begin{array}{l}\text { Oral acy- } \\
\text { clovir }\end{array}$ & $\begin{array}{l}\text { Progressed } \\
\text { to aggressive } \\
\text { NK-cell } \\
\text { leukemia }\end{array}$ & 108 & DOD \\
\hline $5^{9}$ & F & $15(10)$ & $\begin{array}{l}\text { Erythematous } \\
\text { crusted papu- } \\
\text { lovesicles on the } \\
\text { face and arm }\end{array}$ & \begin{tabular}{|l|} 
CD3+, \\
CD45RO+, \\
CD20+ a few, \\
CD56-, Ki-67+ \\
a few, LMP-1-, \\
EBV ISH+, TCR \\
gamma gene \\
arrangement- \\
\end{tabular} & ND & Negative & None & Observation & Remission & 36 & AWD \\
\hline 6 & M & $17(17)$ & $\begin{array}{l}\text { Erythematous } \\
\text { crusted papu- } \\
\text { lovesicles on the } \\
\text { face and trunk, } \\
\text { and oral ulcer }\end{array}$ & $\begin{array}{l}\text { CD3+, CD4+, } \\
\text { CD8+ focal, } \\
\text { CD20-, CD56-, } \\
\text { TIA-1+, Ki-67 } \\
\text { 70\%, EBV ISH+, } \\
\text { TCR gamma } \\
\text { gene rearrange- } \\
\text { ment- }\end{array}$ & $8,463,700$ & ND & Bone marrow & $\begin{array}{l}\text { Systemic } \\
\text { ster- } \\
\text { oid } \rightarrow \text { chem- } \\
\text { otherapy }\end{array}$ & $\begin{array}{l}\text { Progressed } \\
\text { to systemic } \\
\text { EBV+ T-cell } \\
\text { lymphoma }\end{array}$ & 12 & AWD \\
\hline $7^{10}$ & M & $17(8)$ & $\begin{array}{l}\text { Erythema- } \\
\text { tous crusted } \\
\text { papulovesicles } \\
\text { on the face and } \\
\text { forearm }\end{array}$ & $\begin{array}{l}\text { CD45RO+, } \\
\text { CD20-, EBV } \\
\text { ISH+ }\end{array}$ & ND & Negative & $\begin{array}{l}\text { Cervical lymph } \\
\text { node, liver, } \\
\text { spleen }\end{array}$ & $\begin{array}{l}\text { Systemic } \\
\text { steroid, } \\
\text { chemo- } \\
\text { therapy }\end{array}$ & $\begin{array}{l}\text { Progressed } \\
\text { to systemic } \\
\text { EBV+ T-cell } \\
\text { lymphoma }\end{array}$ & NS & AWD \\
\hline 8 & M & $18(6)$ & $\begin{array}{l}\text { Erythematous } \\
\text { crusted papu- } \\
\text { lovesicles on the } \\
\text { face, arm, and } \\
\text { trunk }\end{array}$ & $\begin{array}{l}\text { CD3+, CD4+, } \\
\text { CD8+ focal, } \\
\text { CD20-, CD30+ } \\
\text { a few, CD56+, } \\
\text { granzyme B+, } \\
\text { Ki-67 10\%, EBV } \\
\text { ISH+ }\end{array}$ & $47,925,563$ & Positive & $\begin{array}{l}\text { Cervical lymph } \\
\text { node, spleen, } \\
\text { iliac bone, bone } \\
\text { marrow }\end{array}$ & $\begin{array}{l}\text { Systemic } \\
\text { ster- } \\
\text { oid } \rightarrow \text { chem- } \\
\text { otherapy }\end{array}$ & $\begin{array}{l}\text { Progressed } \\
\text { to systemic } \\
\text { EBV+ T-cell } \\
\text { lymphoma }\end{array}$ & 12 & DOD \\
\hline 9 & $F$ & $19(17)$ & $\begin{array}{l}\text { Erythematous } \\
\text { crusted papu- } \\
\text { lovesicles on the } \\
\text { face and arm, } \\
\text { oral ulcer, and } \\
\text { facial swelling } \\
\end{array}$ & $\begin{array}{l}\text { CD3+, CD4-, } \\
\text { CD8-, CD20-, } \\
\text { CD56-, CD30-, } \\
\text { EBV ISH+ }\end{array}$ & 693,642 & ND & $\begin{array}{l}\text { right breast, lung, } \\
\text { spleen, liver, } \\
\text { pelvic bone, bone } \\
\text { marrow }\end{array}$ & $\begin{array}{l}\text { Systemic } \\
\text { ster- } \\
\text { oid } \rightarrow \text { chem- } \\
\text { otherapy }\end{array}$ & \begin{tabular}{|l|} 
Progressed \\
to extranodal \\
NK/T-cell \\
lymphoma, \\
nasal type
\end{tabular} & 24 & DOD \\
\hline 10 & M & $19(4)$ & $\begin{array}{l}\text { Erythema- } \\
\text { tous crusted } \\
\text { papulovesicles } \\
\text { on the face, } \\
\text { arm, trunk, and } \\
\text { ankle }\end{array}$ & $\begin{array}{l}\text { CD20+ a few, } \\
\text { CD56+ a few, } \\
\text { LMP-1-, EBV } \\
\text { ISH+ }\end{array}$ & 79,396 & ND & None & Observation & Remission & 204 & AWD \\
\hline $11^{10}$ & M & $19(4)$ & $\begin{array}{l}\text { Erythema- } \\
\text { tous crusted } \\
\text { papulovesicles } \\
\text { on the face and } \\
\text { forearm }\end{array}$ & $\begin{array}{l}\text { CD45RO+, } \\
\text { CD20-, EBV } \\
\text { ISH+ }\end{array}$ & ND & Negative & None & $\begin{array}{l}\text { Systemic } \\
\text { steroid, oral } \\
\text { acyclovir }\end{array}$ & $\begin{array}{l}\text { Partial } \\
\text { remission }\end{array}$ & NS & AWD \\
\hline $12^{10}$ & M & $21(19)$ & $\begin{array}{l}\text { Erythematous } \\
\text { crusted papu- } \\
\text { lovesicles on the } \\
\text { face and oral } \\
\text { ulcer }\end{array}$ & $\begin{array}{l}\text { CD45RO+, } \\
\text { CD20-, EBV } \\
\text { ISH+ }\end{array}$ & ND & Negative & $\begin{array}{l}\text { Cervical lymph } \\
\text { node, liver }\end{array}$ & $\begin{array}{l}\text { Systemic } \\
\text { steroid }\end{array}$ & $\begin{array}{l}\text { Progressed } \\
\text { to Systemic } \\
\text { EBV+ T-cell } \\
\text { lymphoma }\end{array}$ & NS & DOD \\
\hline
\end{tabular}




\begin{tabular}{|c|c|c|c|c|c|c|c|c|c|c|c|}
\hline Patient no. & Sex & $\begin{array}{l}\text { Age at } \\
\text { diagnosis, } \\
\text { years (age at } \\
\text { onset, years) }\end{array}$ & $\begin{array}{l}\text { Cutaneous } \\
\text { manifestations }\end{array}$ & \begin{tabular}{|l|} 
IHC, \\
EBER-1 ISH, \\
TCR $\gamma$ gene \\
rearrangement
\end{tabular} & $\begin{array}{l}\text { Serum EBV } \\
\text { DNA load } \\
\text { (copies/mL) }\end{array}$ & $\begin{array}{l}\text { UVA } \\
\text { provocation } \\
\text { test }\end{array}$ & $\begin{array}{l}\text { Extracutaneous } \\
\text { involvement }\end{array}$ & Treatment & $\begin{array}{l}\text { Disease } \\
\text { course }\end{array}$ & $\begin{array}{l}\text { Follow-up } \\
\text { (months) }\end{array}$ & $\begin{array}{l}\text { Last status } \\
\text { during } \\
\text { follow-up }\end{array}$ \\
\hline 13 & M & $24(10)$ & $\begin{array}{l}\text { Erythematous } \\
\text { crusted papu- } \\
\text { lovesicles on the } \\
\text { face, arm, and } \\
\text { trunk }\end{array}$ & \begin{tabular}{|l|} 
CD3+, \\
CD45RO+, \\
CD4+, CD8+, \\
CD20+ a \\
few, CD56-, \\
granzyme B+ a \\
few, Ki-67 1\%, \\
EBV ISH+, TCR \\
gamma gene \\
rearrangement-
\end{tabular} & $1,840,744$ & ND & $\begin{array}{l}\text { Liver, spleen, } \\
\text { bone marrow }\end{array}$ & $\begin{array}{l}\text { Systemic } \\
\text { ster- } \\
\text { oid } \rightarrow \text { chem- } \\
\text { otherapy }\end{array}$ & $\begin{array}{l}\text { Progressed } \\
\text { to Systemic } \\
\text { EBV+ T-cell } \\
\text { lymphoma }\end{array}$ & 120 & DOD \\
\hline $14^{10}$ & $M$ & $24(18)$ & $\begin{array}{l}\text { Erythema- } \\
\text { tous crusted } \\
\text { papulovesicles } \\
\text { on the face and } \\
\text { forearm }\end{array}$ & $\begin{array}{l}\text { CD45RO+, } \\
\text { CD20-, EBV } \\
\text { ISH+ }\end{array}$ & ND & Negative & $\begin{array}{l}\text { Cervical lymph } \\
\text { node, spleen }\end{array}$ & $\begin{array}{l}\text { Systemic } \\
\text { steroid }\end{array}$ & $\begin{array}{l}\text { Progressed } \\
\text { to peripheral } \\
\text { T-cell } \\
\text { lymphoma of } \\
\text { adulthood }\end{array}$ & NS & DOD \\
\hline 15 & M & $26(25)$ & $\begin{array}{l}\text { Erythematous } \\
\text { crusted papu- } \\
\text { lovesicles on } \\
\text { the face }\end{array}$ & \begin{tabular}{|l|} 
CD3+, CD4+, \\
CD8+ focal, \\
CD 20+ a few, \\
CD30+ focal, \\
CD56-, Ki-67 \\
$15 \%$, TCR \\
gamma gene \\
rearrangement+
\end{tabular} & $30,917,751$ & ND & $\begin{array}{l}\text { Cervical and } \\
\text { axillary lymph } \\
\text { node, spleen }\end{array}$ & $\begin{array}{l}\text { Systemic } \\
\text { ster- } \\
\text { oid } \rightarrow \text { chem- } \\
\text { otherapy }\end{array}$ & $\begin{array}{l}\text { Progressed } \\
\text { to peripheral } \\
\text { T-cell } \\
\text { lymphoma of } \\
\text { adulthood }\end{array}$ & 204 & AWD \\
\hline $16^{9}$ & M & $28(22)$ & $\begin{array}{l}\text { Erythematous } \\
\text { crusted papu- } \\
\text { lovesicles on } \\
\text { the face }\end{array}$ & \begin{tabular}{l|} 
CD3+, \\
CD45RO+, \\
CD20+ a few, \\
CD56-, Ki-67+ \\
a few, LMP-1-, \\
EBV ISH+, TCR \\
gene rearrange- \\
ment-
\end{tabular} & ND & Positive & None & Observation & Remission & 36 & AWD \\
\hline $17^{9}$ & $\mathrm{~F}$ & $28(26)$ & $\begin{array}{l}\text { Erythematous } \\
\text { crusted papu- } \\
\text { lovesicles on the } \\
\text { face and facial } \\
\text { swelling }\end{array}$ & \begin{tabular}{|l|} 
CD3+, \\
CD45RO+, \\
CD20+ a few, \\
CD56-, Ki-67 \\
15\%, EBV ISH+, \\
TCR gamma \\
gene rearrange- \\
ment-
\end{tabular} & ND & ND & None & $\begin{array}{l}\text { Systemic } \\
\text { steroid }\end{array}$ & $\begin{array}{l}\text { Partial } \\
\text { remission } \\
\text { and recur- } \\
\text { rence }\end{array}$ & 36 & Death \\
\hline $18^{9}$ & M & $29(25)$ & $\begin{array}{l}\text { Erythematous } \\
\text { crusted papu- } \\
\text { lovesicles on the } \\
\text { face, arm, and } \\
\text { trunk }\end{array}$ & $\begin{array}{l}\text { CD3+, } \\
\text { CD45RO+, } \\
\text { CD20+ a few, } \\
\text { CD56-, Ki-67+ } \\
(<5 \%), \text { EBV } \\
\text { ISH+, TCR } \\
\text { gamma gene } \\
\text { rearrangement- }\end{array}$ & ND & Positive & None & $\begin{array}{l}\text { Systemic } \\
\text { steroid }\end{array}$ & $\begin{array}{l}\text { Partial } \\
\text { remission } \\
\text { and recur- } \\
\text { rence }\end{array}$ & 36 & AWD \\
\hline 19 & $\mathrm{~F}$ & $30(27)$ & $\begin{array}{l}\text { Erythematous } \\
\text { crusted papu- } \\
\text { lovesicles on the } \\
\text { face and oral } \\
\text { ulcer }\end{array}$ & \begin{tabular}{|l|} 
CD3+, \\
CD45RO+, \\
CD20+ a few, \\
CD56-, LMP- \\
$1-$, Ki-67<5\%, \\
EBV ISH+, TCR \\
gamma gene \\
rearrangement+
\end{tabular} & ND & ND & $\begin{array}{l}\text { Cervical and } \\
\text { axillary lymph } \\
\text { node }\end{array}$ & $\begin{array}{l}\text { Chemo- } \\
\text { therapy }\end{array}$ & $\begin{array}{l}\text { Progressed } \\
\text { to or } \\
\text { accompanied } \\
\text { extranodal } \\
\text { NK/T-cell } \\
\text { lymphoma, } \\
\text { nasal type }\end{array}$ & 12 & AWD \\
\hline 20 & M & $32(15)$ & $\begin{array}{l}\text { Erythematous } \\
\text { crusted papu- } \\
\text { lovesicles on the } \\
\text { face and oral } \\
\text { ulcer }\end{array}$ & $\begin{array}{l}\text { CD3+, CD20+ } \\
\text { a few, CD56-, } \\
\text { granzyme B+, } \\
\text { EBV ISH+, } \\
\text { Ki-67 20\% }\end{array}$ & 54,275 & ND & $\begin{array}{l}\text { Palate, bone } \\
\text { marrow }\end{array}$ & $\begin{array}{l}\text { Chemother- } \\
\text { apy, daratu- } \\
\text { mumab }\end{array}$ & \begin{tabular}{|l} 
Progressed \\
to extranodal \\
NK/T-cell \\
lymphoma, \\
nasal type
\end{tabular} & 48 & AWD \\
\hline $21^{11}$ & $F$ & $33(30)$ & $\begin{array}{l}\text { Erythematous } \\
\text { crusted papu- } \\
\text { lovesicles on } \\
\text { the face }\end{array}$ & \begin{tabular}{l|} 
CD3+, \\
CD45RO+, \\
CD20-, CD30-, \\
CD56-, Ki-67 \\
20\%, EBV ISH+, \\
TCR gamma \\
gene rearrange- \\
ment-
\end{tabular} & ND & Positive & $\begin{array}{l}\text { Cervical lymph } \\
\text { node, stomach }\end{array}$ & $\begin{array}{l}\text { Chemo- } \\
\text { therapy }\end{array}$ & $\begin{array}{l}\text { Progressed } \\
\text { to Systemic } \\
\text { EBV+ T-cell } \\
\text { lymphoma }\end{array}$ & 18 & AWD \\
\hline $22^{11}$ & M & $34(33)$ & $\begin{array}{l}\text { Erythematous } \\
\text { crusted papu- } \\
\text { lovesicles on the } \\
\text { face, arm, and } \\
\text { trunk, and oral } \\
\text { ulcer }\end{array}$ & $\begin{array}{l}\text { CD3+, } \\
\text { CD45RO+, } \\
\text { CD20-, CD30-, } \\
\text { CD56-, Ki-67 } \\
\text { 20\%, EBV ISH+, } \\
\text { TCR gamma } \\
\text { gene rearrange- } \\
\text { ment+ }\end{array}$ & ND & ND & Bone marrow & $\begin{array}{l}\text { Chemo- } \\
\text { therapy }\end{array}$ & $\begin{array}{l}\text { Progressed } \\
\text { to Systemic } \\
\text { EBV+ T-cell } \\
\text { lymphoma }\end{array}$ & 24 & AWD \\
\hline
\end{tabular}




\begin{tabular}{|c|c|c|c|c|c|c|c|c|c|c|c|}
\hline Patient no. & Sex & $\begin{array}{l}\text { Age at } \\
\text { diagnosis, } \\
\text { years (age at } \\
\text { onset, years) }\end{array}$ & $\begin{array}{l}\text { Cutaneous } \\
\text { manifestations }\end{array}$ & $\begin{array}{l}\text { IHC, } \\
\text { EBER-1 ISH, } \\
\text { TCR } \gamma \text { gene } \\
\text { rearrangement }\end{array}$ & $\begin{array}{l}\text { Serum EBV } \\
\text { DNA load } \\
\text { (copies/mL) }\end{array}$ & $\begin{array}{l}\text { UVA } \\
\text { provocation } \\
\text { test }\end{array}$ & $\begin{array}{l}\text { Extracutaneous } \\
\text { involvement }\end{array}$ & Treatment & $\begin{array}{l}\text { Disease } \\
\text { course }\end{array}$ & $\begin{array}{l}\text { Follow-up } \\
\text { (months) }\end{array}$ & $\begin{array}{l}\text { Last status } \\
\text { during } \\
\text { follow-up }\end{array}$ \\
\hline $23^{9}$ & M & $37(32)$ & $\begin{array}{l}\text { Erythematous } \\
\text { crusted papu- } \\
\text { lovesicles on the } \\
\text { face, arm, and } \\
\text { trunk }\end{array}$ & $\begin{array}{l}\text { CD3+, } \\
\text { CD45RO+, } \\
\text { CD20+ a few, } \\
\text { CD56-, Ki-67+ } \\
(<5 \%), \text { EBV } \\
\text { ISH+, TCR } \\
\text { gamma gene } \\
\text { rearrangement- }\end{array}$ & ND & ND & $\begin{array}{l}\text { Cervical lymph } \\
\text { node }\end{array}$ & $\begin{array}{l}\text { Chemo- } \\
\text { therapy }\end{array}$ & $\begin{array}{l}\text { Partial } \\
\text { remission } \\
\text { and recur- } \\
\text { rence }\end{array}$ & 36 & AWD \\
\hline 24 & M & $38(38)$ & $\begin{array}{l}\text { Erythematous } \\
\text { crusted papu- } \\
\text { lovesicles on } \\
\text { the face }\end{array}$ & $\begin{array}{l}\text { CD3+, CD4+ } \\
\text { focal, CD8+ } \\
\text { focal, CD20-, } \\
\text { CD56-, } \\
\text { granzyme B+ } \\
\text { focal, Ki-67+, } \\
\text { EBV ISH+, TCR } \\
\text { gamma gene } \\
\text { rearrangement+ }\end{array}$ & 925,529 & ND & $\begin{array}{l}\text { Lung (Hodgkin } \\
\text { lymphoma), } \\
\text { bone marrow, } \\
\text { spleen, bone }\end{array}$ & $\begin{array}{l}\text { Systemic } \\
\text { ster- } \\
\text { oid } \rightarrow \text { chem- } \\
\text { otherapy }\end{array}$ & $\begin{array}{l}\text { Progressed } \\
\text { to EBV+ } \\
\text { Hodgkin } \\
\text { lymphoma }\end{array}$ & 96 & AWD \\
\hline $25^{9}$ & $\mathrm{~F}$ & $39(36)$ & $\begin{array}{l}\text { Erythematous } \\
\text { crusted papu- } \\
\text { lovesicles on the } \\
\text { face, arm, and } \\
\text { trunk }\end{array}$ & $\begin{array}{l}\text { CD3+, } \\
\text { CD45RO+, } \\
\text { CD20+ a few, } \\
\text { CD56-, Ki-67+ } \\
(<5 \%), \text { EBV } \\
\text { ISH+, TCR } \\
\text { gamma gene } \\
\text { rearrangement- }\end{array}$ & ND & Positive & $\begin{array}{l}\text { Cervical lymph } \\
\text { node }\end{array}$ & $\begin{array}{l}\text { Chemo- } \\
\text { therapy }\end{array}$ & $\begin{array}{l}\text { Partial } \\
\text { remission } \\
\text { and recur- } \\
\text { rence }\end{array}$ & 36 & AWD \\
\hline $26^{12}$ & $\mathrm{~F}$ & $70(70)$ & $\begin{array}{l}\text { Erythema- } \\
\text { tous crusted } \\
\text { papulovesicles } \\
\text { on the face, oral } \\
\text { ulcer, and facial } \\
\text { swelling }\end{array}$ & $\begin{array}{l}\text { CD2+, CD3+, } \\
\text { CD4+ a few, } \\
\text { CD5+, CD8+ } \\
\text { focal, CD20-, } \\
\text { CD30-, CD56-, } \\
\text { EBV ISH }\end{array}$ & 200,500 & Negative & None & $\begin{array}{l}\text { Topical } \\
\text { steroid }\end{array}$ & $\begin{array}{l}\text { Partial } \\
\text { remission } \\
\text { and recur- } \\
\text { rence }\end{array}$ & 12 & AWD \\
\hline
\end{tabular}

Table 2. Clinical features and histopathologic findings in patients with hydroa vacciniforme-like lymphoproliferative disease at our institution. $A W D$ alive with the disease, $D O D$ died of disease, EBER-1 ISH EBV-encoded small nonpolyadenylated RNA (EBER)-1 by in situ hybridization, $F$ female, IHC immunohistochemistry, $M$ male, $N D$ not done, NS not specified exact follow-up duration.

for progression and $\mathrm{p}=1.000$ for mortality). We further analyzed these parameters according to whether disease onset occurred in childhood (age $<18$ years) or adulthood (age $\geq 18$ years). There was no difference between the two groups with regard to either progression to lymphoma or disease-related mortality $(\mathrm{p}=1.000$ and $\mathrm{p}=0.645$, respectively). Additionally, we evaluated the effect of the period from disease onset to first diagnosis, comparing patients with a disease duration of $<10$ years and those with a duration of $\geq 10$ years. There were no differences in the rate of progression to lymphoma or disease-related mortality ( $\mathrm{p}=1.000$ and $\mathrm{p}=0.218$, respectively) in relation to disease duration before first diagnosis.

\section{Discussion}

Hydroa vacciniforme is a rare photodermatosis originally described by Bazin ${ }^{14}$. Although the pathogenesis of hydroa vacciniforme is not clearly established, sensitivity to UVB or UVA has been suggested as a pathologic mechanism $^{15}$. In 1996, Cho et al. first suggested an association with EBV and recurrent necrotic papulovesicular eruptions of the face ${ }^{10}$. As most cases with hydroa vacciniforme manifestations have shown the presence of EBV, the question about the existence of classic hydroa vacciniforme without EBV infection remains unresolved ${ }^{16}$. Therefore, Quintanilla-Martinez and Fend suggested renaming the disease as hydroa vacciniforme EBV-associated lymphoproliferative disorder to encompass the broad clinical spectrum ${ }^{16}$.

HVLPD has been given various names, including hydroa vacciniforme-like lymphoma and EBV-associated vesiculopapular eruption on the face. In 2008, the WHO classification introduced $2 \mathrm{~T}$-cell lymphoproliferative disorders associated with EBV in children: hydroa vacciniforme-like lymphoma and systemic EBV+ lymphoproliferative disease of childhood ${ }^{17}$. In 2016 , the 4 th WHO classification reclassified the disease into two groups: HVLPD and systemic EBV+ T-cell lymphoma of childhood (Table 3$)^{18}$.

The EBV-associated T- and NK-cell lymphoproliferative diseases include EBV-associated hemophagocytic lymphohistiocytosis (HLH); CAEBV of T- and NK-cell type; systemic EBV+ T-cell lymphoma of childhood; aggressive NK-cell leukemia; extranodal NK/T-cell lymphoma, nasal type; and primary EBV+ nodal NK/T-cell lymphoma (Table 4) ${ }^{19,20}$. Primary EBV+ nodal NK/T-cell lymphoma is a new provisional entity defined as peripheral T-cell lymphoma with primary nodal presentation without nasal involvement ${ }^{20}$. Although splenomegaly and hepatic involvement can occur in $73 \%$ and $60 \%$ of the patients, skin and gastrointestinal involvement are $\operatorname{rare}^{21}$. EBV-associated HLH is a life-threatening inflammatory disease with symptoms including high fever, cytopenia, hypofibrinogenemia, elevated serum transaminases, hyperbilirubinemia, prolonged prothrombin time, and hyponatremia; it may occur as a primary condition or secondary to other conditions ${ }^{19}$. Secondary EBV-associated HLH may accompany CAEBV of T- and NK-cell type. HVLPD is classified as a cutaneous form of CAEBV of T- and NK-cell type. CAEBV of T- and NK- cell types has a broad range of clinical manifestations from indolent cutaneous form to a more severe systemic form ${ }^{20}$. Aggressive NK-cell leukemia is a fulminant 


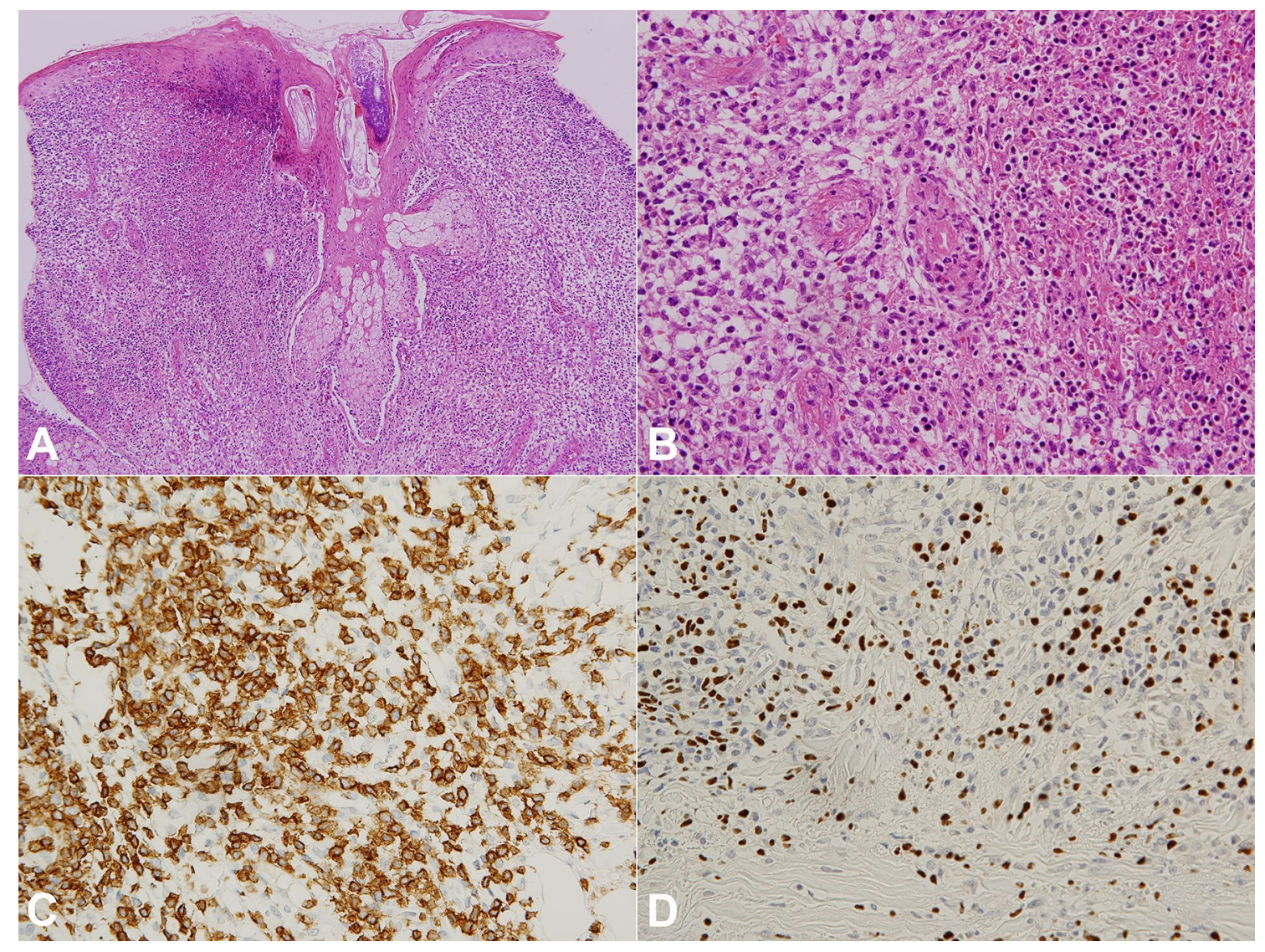

Figure 2. Histopathologic findings of hydroa vacciniforme-like lymphoproliferative disease. (A)

Histopathologic analysis showed diffuse and dense infiltration of lymphocytes in the dermis. Epidermal necrosis, subepidermal edema, and perivascular and periappendageal atypical lymphocytic infiltration were observed (hematoxylin and eosin staining $\times 100$ ). (B) Small- to medium-sized atypical lymphocytes with nuclear hyperchromasia were detected (hematoxylin and eosin staining $\times 400)$. (C) The atypical mononuclear cells were positive for CD3 in immunohistochemical staining $(\times 400)$. (D) In situ hybridization for EBV-encoded small nonpolyadenylated RNA was positive in the atypical mononuclear cells $(\times 400)$.

\begin{tabular}{|c|c|c|}
\hline Diagnosis & Definition & References \\
\hline Hydroa vacciniforme & $\begin{array}{l}\text { Rare, sporadic, idiopathic photodermatosis characterized by papules, vesicles, and crusts } \\
\text { which heal with vacciniform scarring after sunlight exposure. The pathogenesis is unclear } \\
\text { although sensitivity of UVB or UVA has been suggested }\end{array}$ & First reported by Bazin ${ }^{14,15}$ \\
\hline Hydroa vacciniforme-like lymphoma & $\begin{array}{l}\text { Proliferation of clonal } \mathrm{T} \text { cells or, less frequently, NK cells infected by EBV with a latency } \\
\text { type } 1 \text { profile. It has an indolent clinical course with long periods of recurrent skin lesions } \\
\text { in sun-exposed areas that tends to regress spontaneously. After several years, the process } \\
\text { may resolve or progress to systemic disease }\end{array}$ & 2008 WHO classification ${ }^{17}$ \\
\hline Systemic EBV+ lymphoproliferative disease of childhood & $\begin{array}{l}\text { Aggressive condition with rapid evolution to multiple-organ failure and death. It has over- } \\
\text { lapping features with aggressive NK-cell leukemia, but the cells have a T-cell phenotype } \\
\text { and clonal TCR rearrangement. It may emerge in a background of chronic EBV infection } \\
\text { and progress from a polyclonal, to an oligoclonal, and to a monoclonal EBV-driven } \\
\text { proliferation }\end{array}$ & \\
\hline Hydroa vacciniforme-like lymphoproliferative disease & $\begin{array}{l}\text { Name changed from lymphoma to lymphoproliferative disorder due to its relationship } \\
\text { with chronic active EBV infection and the broad spectrum of its clinical course }\end{array}$ & 2016 WHO classification ${ }^{18}$ \\
\hline Systemic EBV+ T-cell lymphoma of childhood & $\begin{array}{l}\text { Name changed from lymphoproliferative disorder to lymphoma owing to its fulminant } \\
\text { clinical course and the desire to clearly distinguish it from chronic active EBV infection }\end{array}$ & \\
\hline
\end{tabular}

Table 3. Change of definition of hydroa vacciniforme and hydroa vacciniforme-like lymphoproliferative disease.

disease characterized by systemic neoplastic proliferation of NK-cells ${ }^{19}$. It occurs in young to middle-aged adults. Extranodal NK/T-cell lymphoma, nasal type, is EBV+ aggressive lymphoma and occurs mainly in middle-aged adults. It occurs in $70-80 \%$ of all cases and primarily involves the nasal and nasopharyngeal region. Skin lesion presents as nodular ulcerative lesions, and gastrointestinal tract, testis and mucous membrane are involved ${ }^{19}$. Systemic EBV+ T-cell lymphoma of childhood is a more rapidly progressive and fatal disease, characterized 


\begin{tabular}{|l|}
\hline Disease \\
\hline Aggressive NK-cell leukemia \\
\hline Chronic active EBV infection (CAEBV) of T- and NK-cell type \\
\hline Cutaneous CAEBV of T- and NK-cell type \\
\hline Hydroa vacciniforme-like lymphoproliferative disease \\
\hline Severe mosquito bite allergy \\
\hline Systemic CAEBV of T- and NK-cell type \\
\hline EBV-associated hemophagocytic lymphohistiocytosis \\
\hline Extranodal NK/T-cell lymphoma, nasal type \\
\hline Primary EBV+ nodal NK/T-cell lymphoma \\
\hline Systemic EBV+ T-cell lymphoma of childhood \\
\hline
\end{tabular}

Table 4. Classification of EBV-associated T- and NK-cell lymphoproliferative diseases ${ }^{19,20}$. ${ }^{\mathrm{a}} \mathrm{A}$ provisional entity.

by fever, hepatosplenomegaly, liver failure, lymphadenopathy, HLH, and pancytopenia ${ }^{1,20}$. However, as some features overlap among EBV-associated T- and NK-cell lymphoproliferative diseases, the distinction of diseases is sometimes challenging.

HVLPD occurs in the presence of chronic EBV infection and presents characteristic sun-related skin eruptions. Although patients with HVLPD can show a favorable prognosis, some patients may have significantly serious outcomes, including progression to systemic lymphoma. These cases with extracutaneous involvement of atypical lesional cells are diagnosed as systemic EBV+ T-cell lymphoma of childhood according to the revised 4 th WHO classification ${ }^{1,2}$. Although classic HVLPD usually occurs in the pediatric age group, there have been cases of systemic EBV+ T-cell lymphoma in adults with HVLPD ${ }^{3,5,22}$.

The prognostic factors of progression from HVLPD to systemic lymphoma have not been definitely established. Factors such as high serum EBV DNA loads, Latin American descent, systemic symptoms, adult onset, and chemotherapy were suggested to be associated with a more aggressive disease course $e^{3,10,19,20}$. In our database, among 19 adult cases, 11 (57.9\%) were associated with systemic lymphoma, and this proportion was higher than that observed among childhood cases. Among these cases, seven progressed to systemic EBV+ T-cell lymphoma; three to extranodal NK/T-cell lymphoma, nasal type; and one to EBV+ Hodgkin lymphoma. Therefore, considering the higher possibility of adverse outcomes, adult-onset cases may also need careful monitoring as in children cases.

There is no established treatment guideline for HVLPD. Stringent sun protection is recommended for all patients using broad-spectrum sunscreens, sun-protecting clothing, and avoidance of sun exposure. Various treatments were tried including hydroxychloroquine, anti-viral drugs (acyclovir and valacyclovir), topical and systemic corticosteroids, and chemotherapy. For systemic HVLPD, although corticosteroids or thalidomide provided temporary improvements, the only curative treatment is hematopoietic stem cell transplantation ${ }^{23}$. A recent report suggested that chemotherapy should not be considered as the first-line treatment as it was associated with poor prognosis in patients with $\mathrm{HVLPD}^{3}$. However, chemotherapy may be an effective method to reduce systemic disease burden in an effort to enhance the curative potential of allogeneic bone marrow transplantation, with or without the reduction of EBV viral load ${ }^{24}$. Therefore, its use should be prudently considered in selected cases. Further investigations on which patients can benefit from chemotherapy are warranted.

There are some limitations. Although this study included one of the large cases with three decades data in SNUH, the sample size is small as HVLPD is an extremely rare disease. In addition, a detailed molecular analysis was not performed due to the retrospective study design. Lastly, it was challenging to distinguish between HVLPD and extranodal NK/T-cell lymphoma, nasal type, and primary EBV+ nodal T/NK-cell lymphoma with extranodal involvement as some features are overlapped. However, the diagnosis was made based on clinical and pathologic findings by an experienced dermatologist, pathologist, and oncologist.

In summary, we described 26 cases of HVLPD encountered at our institution during the last three decades. HVLPD seems to have various disease courses. Fourteen patients $(53.8 \%)$ in the present study had systemic T-cell lymphoma, NK-T cell lymphoma/leukemia, and EBV+ Hodgkin lymphoma. Lymphoma progression and mortality can occur not only in childhood but also in adulthood.

\section{Methods}

We retrospectively reviewed cases of HVLPD diagnosed based on clinical and histopathologic features between 1988 and 2019 in the Department of Dermatology of SNUH. Patients who both gave and signed an informed consent to perform a skin biopsy were recruited (In cases of patients under 18 years of age, signed informed consent were given by parents and/or legal guardians). HVLPD was defined according to the revised 4th WHO classification of hematopoietic and lymphoid tumors ${ }^{1}$. Clinical and laboratory data the time of diagnosis were obtained from medical records.

Histopathologic features, immunohistochemical findings, EBER by in situ hybridization, serum EBV loads, T-cell clonality test using multiplex-PCR for the T-cell receptor $\gamma$ gene, and results of radiologic imaging studies were reviewed. Photoprovocation tests UVA. A $5 \times 5 \mathrm{~cm}$ area on the buttock or upper arm was irradiated 3 or 5 times at $24-\mathrm{h}$ intervals $\left(15-20 \mathrm{~J} / \mathrm{cm}^{2}\right)^{9}$. This study was approved by the institutional review board of 
SNUH (approval no. 1908-019-1053). All methods were carried out in accordance with relevant guidelines and regulations.

Statistical analyses were performed using SPSS 25 (SPSS Inc., IL, USA). Pearson's chi-square test was performed to compare proportions, except when the expected values in the SPSS cells were $<5$, in which case Fisher's exact test was used. Student's t-test was used to compare mean disease durations.

Received: 6 May 2020; Accepted: 27 October 2020

Published online: 09 November 2020

\section{References}

1. Swerdlow, S. H. et al. The 2016 revision of the World Health Organization classification of lymphoid neoplasms. Blood 127, 2375-2390 (2016).

2. Sundram, U. Cutaneous lymphoproliferative disorders: What's new in the revised 4th edition of the World Health Organization (WHO) classification of lymphoid neoplasms. Adv. Anat. Pathol. 26, 93-113 (2019).

3. Liu, Y., Ma, C., Wang, G. \& Wang, L. Hydroa vacciniforme-like lymphoproliferative disorder: Clinicopathologic study of 41 cases. J. Am. Acad. Dermatol. 81, 534-540 (2019).

4. Lyapichev, K. A., Sukswai, N., Wang, X. I., Khoury, J. D. \& Medeiros, L. J. Hydroa vacciniforme-like lymphoproliferative disorder with progression to EBV+ cytotoxic peripheral T-cell lymphoma. Am. J. Dermatopathol. https://doi.org/10.1097/DAD.0000000000 001494 (2019) (Epub ahead of print).

5. Wen, P. F. et al. Comparative study of the clinical pathology, immunophenotype, Epstein-Barr virus infection status, and gene rearrangements in adult and child patients with hydroa vacciniforme-like lymphoproliferative disorder. Am. J. Dermatopathol. 41, 7-15 (2019).

6. Kai, K. et al. Autopsy case of systemic EBV-positive T-cell lymphoma of childhood with marked hepatomegaly in a middle-aged man. Pathol. Int. 67, 431-433 (2017).

7. Huh, S. Y., Choi, M. \& Cho, K. H. A case of Epstein-Barr virus-associated hydroa vacciniforme. Ann. Dermatol. 21, 209-212 (2009).

8. Cho, K. H. et al. Epstein-Barr virus-associated lymphoproliferative eruption with progression to large granular lymphocytic leukaemia. Br. J. Dermatol. 137, 426-430 (1997).

9. Cho, K. H. et al. Epstein-Barr virus-associated lymphoproliferative lesions presenting as a hydroa vacciniforme-like eruption: an analysis of six cases. Br. J. Dermatol. 151, 372-380 (2004).

10. Cho, K. H. et al. An Epstein-Barr virus-associated lymphoproliferative lesion of the skin presenting as recurrent necrotic papulovesicles of the face. Br. J. Dermatol. 134, 791-796 (1996).

11. Cho, K. H. et al. Epstein-Barr virus-associated peripheral T-cell lymphoma in adults with hydroa vacciniforme-like lesions. Clin. Exp. Dermatol. 26, 242-247 (2001).

12. Jung, S. E., Cho, K. H., Lee, M. W. \& Kim, Y. C. Hydroa vacciniforme-like eruption associated with Epstein-Barr virus infection in an older adult. Ann. Dermatol. 27, 789-791 (2015).

13. Paik, J. H. et al. Clinicopathological categorization of Epstein-Barr virus-positive T/NK-cell lymphoproliferative disease: An analysis of 42 cases with an emphasis on prognostic implications. Leuk. Lymphoma. 58, 53-63 (2017).

14. Bazin E. Leçons théoriques et cliniques sur les affections génériques de la peau. (Adrien Delahaye, 1862).

15. Gupta, G., Man, I. \& Kemmett, D. Hydroa vacciniforme: A clinical and follow-up study of 17 cases. J. Am. Acad. Dermatol. 42, 208-213 (2000).

16. Quintanilla-Martinez, L. \& Fend, F. Deciphering hydroa vacciniforme. Blood 133, 2735-2737 (2019).

17. Campo, E. et al. The 2008 WHO classification of lymphoid neoplasms and beyond: Evolving concepts and practical applications. Blood 117, 5019-5032 (2011).

18. Arber, D. A. et al. The 2016 revision to the World Health Organization classification of myeloid neoplasms and acute leukemia. Blood 127, 2391-2405 (2016).

19. Kim, W. Y., Montes-Mojarro, I. A., Fend, F. \& Quintanilla-Martinez, L. Epstein-Barr virus-associated T and NK-cell lymphoproliferative diseases. Front. Pediatr. 7, 71 (2019).

20. Cohen, J. I. et al. Epstein-Barr virus NK and T cell lymphoproliferative disease: Report of a 2018 international meeting. Leuk. Lymphoma. 61, 808-819 (2020).

21. Jeon, Y. K., Kim, J. H., Sung, J. Y., Han, J. H. \& Ko, Y. H. Epstein-Barr virus-positive nodal T/NK-cell lymphoma: An analysis of 15 cases with distinct clinicopathological features. Hum. Pathol. 46, 981-990 (2015).

22. Long, V., Liang, M. W. \& Tan, S. H. Hydroa vacciniforme-like lymphoproliferative disorder in an elderly Chinese patient and a literature review of adult cases. Int. J. Dermatol. 57, 1283-1292 (2018).

23. Xue, R. et al. Hydroa vacciniforme-like lymphoma: Clinicopathological description, treatment and outcome. J. Am. Acad. Dermatol. Preprint at https://doi.org/10.1016/j.jaad.2019.11.031 (2019).

24. Cohen, J. I. et al. Hydroa vacciniforme-like lymphoproliferative disorder: An EBV disease with a low risk of systemic illness in whites. Blood 133, 2753-2764 (2019).

\section{Acknowledgements}

We are grateful to Dr. Kwang Hyun Cho for his dedication to the understanding of Epstein-Barr virus-associated vesiculopapular eruption on the face/hydroa vacciniforme-like lymphoproliferative disease. We also thank all HVLPD patients.

\section{Author contributions}

Conceptualization, B.H. and J.H.M.; methodology, B.H. and J.H.M.; validation, K.H. and J.O.; formal analysis, B.H.; investigation, B.H., K.H., J.O. and J.H.M.; resources, Y.C.K and J.H.M.; data curation, B.H., K.H., J.O., T.M.K., Y.K.J., Y.C.K., and J.H.M.; writing-original draft preparation, B.H.; writing-review and editing, K.H., J.O., T.M.K., Y.K.J., Y.C.K., and J.H.M.; supervision, J.H.M. All authors have read and agreed to the published version of the manuscript.

\section{Funding}

This research received no external funding. 


\section{Competing interests}

The authors declare no competing interests.

\section{Additional information}

Correspondence and requests for materials should be addressed to J.-H.M.

Reprints and permissions information is available at www.nature.com/reprints.

Publisher's note Springer Nature remains neutral with regard to jurisdictional claims in published maps and institutional affiliations.

(c) (1) Open Access This article is licensed under a Creative Commons Attribution 4.0 International License, which permits use, sharing, adaptation, distribution and reproduction in any medium or format, as long as you give appropriate credit to the original author(s) and the source, provide a link to the Creative Commons licence, and indicate if changes were made. The images or other third party material in this article are included in the article's Creative Commons licence, unless indicated otherwise in a credit line to the material. If material is not included in the article's Creative Commons licence and your intended use is not permitted by statutory regulation or exceeds the permitted use, you will need to obtain permission directly from the copyright holder. To view a copy of this licence, visit http://creativecommons.org/licenses/by/4.0/.

(c) The Author(s) 2020 